\title{
Phenomenological friction equation for turbulent flow of Bingham fluids
}

\author{
H. R. Anbarlooei, ${ }^{1}$ D. O. A. Cruz,${ }^{1, *}$ F. Ramos,${ }^{2, \dagger}$ Cecilia M. M. Santos,${ }^{1}$ and A. P. Silva Freire ${ }^{1}$ \\ ${ }^{1}$ Mechanical Engineering Program, COPPE, Federal University of Rio de Janeiro, Rio de Janeiro, Rio de Janeiro 21941-450, Brazil \\ ${ }^{2}$ Department of Applied Mathematics, Institute of Mathematics, Federal University of Rio de Janeiro, Rio de Janeiro, Rio de Janeiro \\ 21941-450, Brazil
}

(Received 1 November 2016; revised manuscript received 16 March 2017; published 25 August 2017)

\begin{abstract}
Most discussions in the literature on the friction coefficient of turbulent flows of fluids with complex rheology are empirical. As a rule, theoretical frameworks are not available even for some relatively simple constitutive models. In the present work, a formula is proposed for the evaluation of the friction coefficient of turbulent flows of Bingham fluids. The developments combine a fresh analysis for the description of the microscales of Kolmogorov and the phenomenological turbulence model of Gioia and Chakraborty [G. Gioia and P. Chakraborty, Phys. Rev. Lett. 96, 044502 (2006)]. The resulting Blasius-type friction equation is tested against some experimental data and shows good agreement over a significant range of Hedstrom and Reynolds numbers. Comments on pressure measurements in yielding fluids are made. The limits of the proposed model are also discussed.
\end{abstract}

DOI: 10.1103/PhysRevE.96.023107

\section{INTRODUCTION}

The seminal works of Prandtl and Blasius in the beginning of the 20th century produced an answer for the determination of the drag or resistance of external and internal flows. The formulations were a great advance over the trivial solutions provided by the 19th century Eulerian (inviscid) hydrodynamics, furnishing in particular consistent solutions to the common problem of estimating the pressure loss suffered by a liquid flowing around a flat plate or through a pipe. The approaches basically handled common fluids such as air and water; more complex fluids were left for particular consideration.

Viscoplastic fluids are a distinguished class of complex fluids, for they exhibit a dual response to shear stresses: At low stresses their behavior resembles that of a rigid solid; at high stresses they flow like a fluid. The most common idealization of a viscoplastic fluid is the Bingham model. In this model, a sharp yield stress value $\tau_{y}$ exists such that for an instantaneous shear stress $\tau<\tau_{y}$, the local deformation rate $\dot{\gamma}$ is null; for $\tau>\tau_{y}$, a Newtonian fluid flow is triggered in which stress and strain are linearly related through a constant plastic viscosity $\mu_{b}$. The mathematical description of the Bingham fluid is

$$
\dot{\gamma}= \begin{cases}0 & \text { for } \tau<\tau_{y} \\ \mu_{b}^{-1}\left(\tau-\tau_{y}\right) & \text { otherwise. }\end{cases}
$$

The viscous effects of the wall vicinity on the mean axial momentum are normally encapsulated into a single nondimensional number, the Darcy friction factor $C_{f}=8 \tau_{w} / \rho U^{2}$, where $U$ is a characteristic velocity of the flow (usually the mean velocity), $\rho$ is the density of the fluid, and $\tau_{w}$ is the wall shear stress, a measure of force per unit area exerted by the fluid on the wall surface.

In [1], the empirical resistance law of Blasius for Newtonian fluids suggests $C_{f} \sim 1 / \operatorname{Re}^{1 / 4}$, in what has been proven to be a good approximation for turbulent flows with Reynolds number up to a few hundred thousands. Nikuradse [2] further showed

\footnotetext{
*doac@mecanica.coppe.ufrj.br

†framos@ufrj.br
}

that in a rough pipe, the friction also depends on the relative roughness size $\varepsilon / R$.

The task of finding an appropriate relation for the ratio between inertial and viscous forces is a complex subject for non-Newtonian fluid flows. For power-law flows, Dodge and Metzner [3] proposed a Reynolds-number formulation that is able to combine all the non-Newtonian parameters into one single expression. In the presence of yielding stresses, the progress has been, however, elusive.

For Bingham fluid flows, the appropriate Reynolds-number definition depends only on the Bingham viscosity. Nevertheless, the presence of the yield stress parameter gives rise to an additional nondimensional parameter, the Hedstrom number $\mathrm{He}$, which is a measure of the magnitude of the yield stress effects $\tau_{y}$ relative to the dynamic Bingham viscosity $\mu_{b}$ :

$$
\operatorname{Re}_{b}=\frac{\rho U L}{\mu_{b}}, \quad \mathrm{He}=\frac{\rho \tau_{y} L^{2}}{\mu_{b}^{2}} .
$$

In [4], Darby and Melson proposed a semiempirical expression for the Fanning friction factor of fully developed turbulent flow of Bingham fluid, which reads

$$
C_{f}=10^{a} \operatorname{Re}_{b}^{-0.193}
$$

where $a=-1.47\left[1+0.146 \exp \left(-2.9 \times 10^{-5} \mathrm{He}\right)\right]$.

In the present work, an alternative friction relation is proposed for Bingham fluids as the result of an analysis based on two fundamental premises: (i) the dimensional arguments of Kolmogorov and (ii) a phenomenological closure model for the Reynolds' stresses first proposed in [5] for Newtonian flows in the presence of rough walls. This methodology has been fundamental to the developments presented in [6], where an analytical friction coefficient for power-law fluid flows was proposed. However, this extension is not straightforward for the viscoplastic materials and requires further considerations about the energy cascade and energy spectrum.

The diversity of complex fluids and their rheological behavior naturally hampers the advancement of simple classification rules and universal mathematical frameworks. The traditional attempt at classifying complex fluids into two groups (Newtonian or Non-Newtonian fluids) has proven to 
be ineffective, since the term "non-Newtonian" contains a large family of fluids with unrelated physical behavior. A common classification consists in splitting non-Newtonian fluids into three essential groups: purely viscous, viscoplastic, and viscoelastic. In [6], the power-law model was considered to propose a friction factor equation. The resulting equation was developed as a function of the generalized Metzner-Reed Reynolds number $\mathrm{Re}_{\mathrm{MR}}$, which encapsulates in one single expression the ratio between inertial and rheological stresses.

Viscoplastic fluids are in unambiguous contrast to the power-law model, which, despite its variable effective viscosity, does not present a sharp behavior at slightly different stresses. This feature gives rise to an additional nondimensional parameter, the Hedstrom number He [Eq. (2)], which is a measure of the magnitude of the yield stress effects relative to the dynamic Bingham viscosity.

From the modeling point of view, the main difference between the results of [6] and the present results is that the Kolmogorov transitional scales, the wall units, and the friction velocity are now obtained as a function of two nondimensional control parameters $\mathrm{Re}$ and He. The latter control parameter leads to a different description of Kolmogorov's cascade scenario in terms of the stress tensors, which needs to incorporate the additional yielding stress. The resulting equation for the viscous stress is shown here to be an explicit secondorder polynomial relation, a nontrivial relation between the Kolmogorov and the macroscopic velocity scales as a function of both $\mathrm{Re}$ and He. The proposed friction factor expression is described by a family of curves, one for every fixed value of He. Another important difference concerns the limits of validity of the Blasius-like expressions. Here we have a two-dimensional region of validity that agrees well with experiments. This is in contrast to the friction equation obtained for Newtonian fluids and power-law fluids that can be described by a single curve, with limits of validity described by a single interval. The derivation of Kolmogorov's and wall length scales in the presence of multiple nondimensional dynamic control parameters is important to the study of turbulence of other complex and relevant fluid flows, such as viscoelastic fluids, where multiple nondimensional dynamic control parameters are present, such as the Reynolds, Weissenberg, and Deborah numbers.

The structure of the paper is as follows. In Sec. II A key assumptions and consequences of Kolmogorov's theory are reviewed. The extension to non-Newtonian Bingham fluid is described in Sec. II B. The phenomenological turbulent model and the resulting friction equation are discussed in Sec. III. Considerations of the limits of validity of the proposed equation are introduced in Sec. IV. A summary is given in Sec. V.

\section{KOLMOGOROV'S SCALING}

\section{A. Newtonian fluids}

Kolmogorov published the first of a series of ground breaking articles concerning the investigation of fully developed turbulent Newtonian fluid flows [7]. These works were originally formulated on purely dimensional grounds and based on Richardson's energy cascade scenario. The latter concept argues that in a turbulent fluid flow in statistical equilibrium, where the motion is mainly driven by forces acting at large scales, energy cascades through intermediate scales, the socalled inertial range, from larger to small scales, where it gets dissipated. The main assumption advanced by Kolmogorov is that in the inertial range, the average energy flux is constant, independent of the kinematic viscosity $v$, and equals the mean energy dissipation rate per unit mass $\epsilon$ (see [8,9]). We now derive some of the well known Kolmogorov relations through scaling arguments for the related stress tensors, since using this unconventional quantity (stress rather than strain rate) is more suited for working with non-Newtonian fluids.

First, we define an inertial stress at scale $\ell$ as $\tau_{\ell} \sim \rho u_{\ell}^{2}$, where $u_{\ell}$ is the characteristic velocity of typical eddies at scale $\ell$. We can also define the characteristic time scale $\ell$ as $t_{\ell} \sim \ell / u_{\ell}$. Now, assuming that the eddy with characteristic scale $\ell$ lies in the inertial range, where the energy dissipation rate by unit mass is constant and equals $\epsilon$, it is argued on dimensional grounds that the inertial stress in units of time at scale $\ell$ must balance the energy dissipation rate balance so that $\tau_{\ell} / t_{\ell} \sim \tau_{\ell} /\left(\ell / u_{\ell}\right) \sim \rho \epsilon$. This leads to the celebrated Kolmogorov spectral relation $u_{\ell} \sim \epsilon^{1 / 3} \ell^{1 / 3}$. From this relation, we can rewrite the inertial strain rate relation at scale $\ell$ as $\tau_{\ell} \sim \rho \epsilon^{2 / 3} \ell^{2 / 3}$ for eddies in the inertial range. This phenomenology is independent of the rheology of the considered fluid and thus also justifiable for fluid flows described by the Bingham model.

Kolmogorov's phenomenology implies the existence of a transitional scale $\ell_{d}$, called Kolmogorov's dissipative scale, between the inertial and dissipative ranges, where both viscous and inertial effects are important. At this transitional scale, the energy relation $\rho \epsilon \sim \rho \epsilon_{d} \sim \mu u_{\ell_{d}}^{2} / \ell_{d}^{2}$ holds, which follows from the energy equation of Newtonian flows. From this last relation, one may define a viscous stress at scale $\ell$ as $\tau_{\mu} \sim \mu u_{\ell} / \ell \sim(\mu \rho \epsilon)^{1 / 2}$. Because $\tau_{\mu} \sim \tau_{\ell}$ at scale $\ell_{d}$, we recover Kolmogorov's dissipative scale $\ell_{d} \sim\left(\mu^{3} / \rho^{3} \epsilon\right)^{1 / 4}$. The scale $\ell_{d}$ depends of course on the rheology of the fluid, so Kolmogorov's scale must be modified for Bingham flows as discussed in the following section. A discussion of changes in the Kolmogorov scale for power-law fluids is presented in [6].

The relation between Kolmogorov's scale $\ell_{d}$ and the characteristic macroscale $L$ is obtained through the following arguments. At the top of the inertial range, the global energy flux relation $\epsilon \sim U^{3} / L$ holds. Inserting this relation into the relation for $\ell_{d}$ results in $\ell_{d} / L \sim 1 / \mathrm{Re}^{3 / 4}$. The arguments also imply the relation $u_{d} / U \sim\left(\ell_{d} / L\right)^{1 / 3} \sim 1 / \operatorname{Re}^{1 / 4}$. As reported in [6], this last relation is crucial for the derivation of a phenomenological friction factor equation for power-law fluid flows. Equally, the same arguments are crucial for the developments of the present work.

Kolmogorov's spectral relations were originally formulated for Newtonian flows, but are observed in several experiments with turbulent non-Newtonian fluid flows. The relations hold even in an anisotropic context, where the presence of other physical scalings challenges the assumption of the existence of an inertial range, in the spirit of Landau's famous criticisms of Kolmogorov's theory (see [5,6,10]).

\section{B. Turbulent Bingham fluids}

We now describe how the Kolmogorov phenomenology can be recovered for turbulent Bingham fluid flows. First, it is well 
known that the central plug disappears at the end of the laminar to turbulent transition in the yielding fluids [11-14]. Due to lack of observation of any microplug region in the turbulent flow, the researchers argue that the local stress is always higher than the yielding limit of the fluid in this regime [11,12]. A more fundamental explanation is also offered in [13] based on the interaction between the turbulence and the internal structure of viscoplastic fluids. In this work, the authors argue that the process of cross-link formation and destruction is not instantaneous and yielding fluids show extensive thixotropic properties. The instantaneous zero shear regions do not act like a solid immediately but need time to relax to the behavior of a solid. However, the turbulent time scales are shorter than the relaxation time, so microplug regions cannot form in turbulent flows [13].

To obtain the dissipative scales, the dimensional analysis for the viscous strain rate relation needs to be modified. Consideration of either the first or the last reasoning implies that the constitutive equation (1) can be simplified by assuming that eventual microplugs do not interfere with the energy cascade mechanism. For Bingham flows, at the transitional scale $\ell_{d}$, the energy dissipation relation becomes

$$
\rho \epsilon \sim \rho \epsilon_{d} \sim\left(\tau_{y}+\mu_{b} \frac{u_{d}}{\ell_{d}}\right) \frac{u_{d}}{\ell_{d}} .
$$

Multiplying Eq. (4) by $\mu_{b}$, we obtain a second-order degree polynomial for the characteristic viscous stress at the transitional scale $\tau_{\mu, d}=\mu_{b} u_{d} / \ell_{d}$,

$$
\tau_{\mu, d}^{2}+\tau_{y} \tau_{\mu, d}-\rho \mu_{b} \epsilon=0
$$

with the positive solution

$$
\tau_{\mu, d}=\frac{\sqrt{\tau_{y}^{2}+4 \rho \epsilon \mu_{b}}-\tau_{y}}{2} .
$$

In the transitional scale, because the flow is in statistical equilibrium, dimensional reasoning leads to the following balance relation between the inertial stress and the total dissipative stress $\tau_{d}$ :

$$
\rho u_{d}^{2} \sim \tau_{d} \equiv \tau_{y}+\tau_{\mu, d} .
$$

Thus, we obtain

$$
u_{d} \sim \sqrt{\frac{\tau_{y}+\tau_{\mu, d}}{\rho}}=\sqrt{\frac{\tau_{y}+\sqrt{\tau_{y}^{2}+4 \rho \epsilon \mu_{b}}}{2 \rho}} .
$$

This statement is equivalent to the following local Reynolds number relation at the dissipative scale:

$$
\operatorname{Re}_{d}=\frac{\rho u_{d} \ell_{d}}{\mu_{e}}=\frac{\rho u_{d}^{2}}{\mu_{b} t_{d}^{-1}+\tau_{y}}=1 .
$$

Equation (8) furnishes immediately

$$
\frac{u_{d}}{U} \sim \sqrt{\frac{\frac{\tau_{y}}{\rho U^{2}}+\sqrt{\left(\tau_{y} / \rho U^{2}\right)^{2}+4 \rho \epsilon \mu_{b} / \rho^{2} U^{4}}}{2}} .
$$

Since $\tau_{y} / \rho U^{2}=\mathrm{He} / \operatorname{Re}^{2}$ and $1 / \operatorname{Re}=\rho \epsilon \mu_{b} / \rho^{2} U^{4}$, it follows that

$$
\frac{u_{d}}{U} \sim \frac{1}{\sqrt{2}} \sqrt{\sqrt{\frac{\mathrm{He}^{2}}{\mathrm{Re}^{4}}+\frac{4}{\mathrm{Re}}}+\frac{\mathrm{He}}{\mathrm{Re}^{2}}} .
$$

\section{PHENOMENOLOGICAL FRICTION FACTOR}

In [6], a friction factor relation for power-law fluid flows was derived through a phenomenological closure model for the Reynolds stress tensor first introduced by Gioia and Chakaborty in [5] in the context of Newtonian flows over rough walls. The same approach is used next to model the Reynolds stress tensor of Bingham fluid flows bounded by smooth walls.

The phenomenology considers that for a moderately large Reynolds number, there exists a viscous wet surface $\mathcal{W}$ of constant thickness in the flow, parallel to the wall, such that above it the horizontal velocity of the flow scales as $\sim U$. In this upper region, the fluid flow carries a high horizontal momentum per unit volume $\rho U$. Below $\mathcal{W}$, the velocity of the flow is small and the fluid has a negligible horizontal momentum per unit volume. Consider also that over the wet surface $\mathcal{W}$, the stresses are mainly induced by vertical fluctuations of horizontal momentum, so the net turbulent stresses are dominant over $\mathcal{W}$. Below $\mathcal{W}$, the turbulent stress contribution decays fast, so in the immediate vicinity of the wall, the main contribution to the stress is due to viscous forces.

The analysis proceeds with a pictorial description of eddies that straddle the wet surface $\mathcal{W}$. The eddies transport portions of fluid of high horizontal momentum across $\mathcal{W}$ into the wall direction, thus resulting in portions of fluid with negligible horizontal momentum across $\mathcal{W}$ in the centerline direction. The vortical contribution to the vertical momentum transport across $\mathcal{W}$ is set by $v_{N}$, the eddy's velocity normal to $\mathcal{W}$. As in $[5,6]$, the key hypothesis is that $v_{N} \sim u_{d}$.

Now, because viscous stress is dominated by the inertial vortical fluctuations over $\mathcal{W}$, we assume $\left.\tau\right|_{\mathcal{W}} \sim \rho U u_{d}$. This stress is then balanced by the wall shear stress. The wall shear stress can therefore be stated through our phenomenological picture as the balance law

$$
\tau_{w} \sim \tau_{\mid \mathcal{W}} \sim \rho U u_{d}
$$

so the following friction formula can be advanced:

$$
C_{f}=8 \frac{\tau_{w}}{\rho U^{2}} \sim 8 \frac{\rho U u_{d}}{\rho U^{2}}=g(\mathrm{He}) \frac{u_{d}}{U},
$$

where simple dimensional arguments show that the nondimensional parameter $g(\mathrm{He})$ is dependent only on the parameter He. The effects of the yield stress are known to vanish at high Reynolds number. Inspection of some empirical results suggest that $g$ is not a strong function of He over a relevant range of Reynolds number. In particular, the relation $g(\mathrm{He}) \equiv 0.316$ can be adopted, so the present results comply with Blasius's law for Newtonian fluid flows. 
TABLE I. Comparison of wall shear stress calculated from pressure measurement and velocimetry using experimental results of [14].

\begin{tabular}{lccc}
\hline \hline$\tau_{w}^{F}$ & $\tau_{w}$ & $\tau_{w}^{F} / \tau_{w}$ & $\left(\tau_{w}^{F}+\tau_{y}\right) / \tau_{w}$ \\
\hline 4.54 & 7.80 & 0.58 & 1.14 \\
7.66 & 12.02 & 0.64 & 1.00 \\
10.9 & 15.78 & 0.69 & 0.98 \\
\hline \hline
\end{tabular}

Inserting (11) into (13), the total friction coefficient equation is obtained

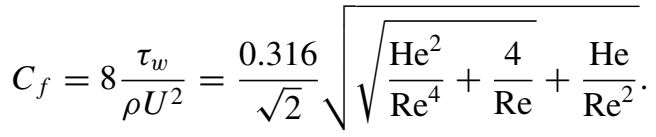

However, as the above expression is compared with the available experimental data for turbulent flow of viscous plastic fluids, the predictions are shown to overestimate the observed results. A close examination of this discrepancy shows that a simple constant shift in Eq. (14) (with equal value of the yield stress) results in a very good match between the two sets (see Fig. 2). This result, of course, suggests the following analysis. The friction factor estimated in controlled experimental simulators is based on pressure drop readings along pipes and channels. However, the measured pressure drop (at least in the turbulent regime) accounts only for the viscous contribution $\mu_{b} d U /\left.d y\right|_{y=0}$. The normal stresses due to the yielding component are not captured by the pressure taps. As a direct consequence, Eq. (14) overestimates the measured pressure drop, since it considers the total wall shear stress.

To further support the above argument, the results of the experimental investigation of turbulent flow of yielding fluids in [14] are reconsidered here. In this study, pressure drop data are available and the velocity field is characterized in a region very close to the wall (with profiles obtained through highresolution laser Doppler anemometry). As it turns out, a mismatch between wall shear stress predictions obtained directly from pressure drop measurements $\left[\tau_{w}^{F}=(R / 2)(d p / d x)\right]$ and from the linear near wall velocity profile $\left(\tau_{w}=\tau_{y}+\mu_{b} \dot{\gamma}\right)$ is apparent (Table I). The discrepancy was justified by the authors as a mere consequence of the thixotropic nature of the investigated fluid. However, a thorough analysis of these experimental results indicates that the difference between the two evaluated wall shear stresses is identical to the yield stress, as indicated by $\tau_{w}-\tau_{w}^{F}=\tau_{y}$ (see Table I).

Similar discrepancies between measured and expected pressure drops, and the nature of pressure transmission for Bingham flows have been discussed before in the literature, as pointed out in [15] and references therein. Whether the source of this anomalous behavior lies in the techniques used to measure the pressure or is a more fundamental issue cannot be concluded at this stage and is left as an open question for future studies.

Hence, to compare our phenomenological friction factor with the measured friction coefficient, the term $\tau_{y}$ needs to be subtracted from the definition of the wall shear stress in Eq. (14) so that one must consider $\tau_{w}^{F}=\tau_{w}-\tau_{y}$. This

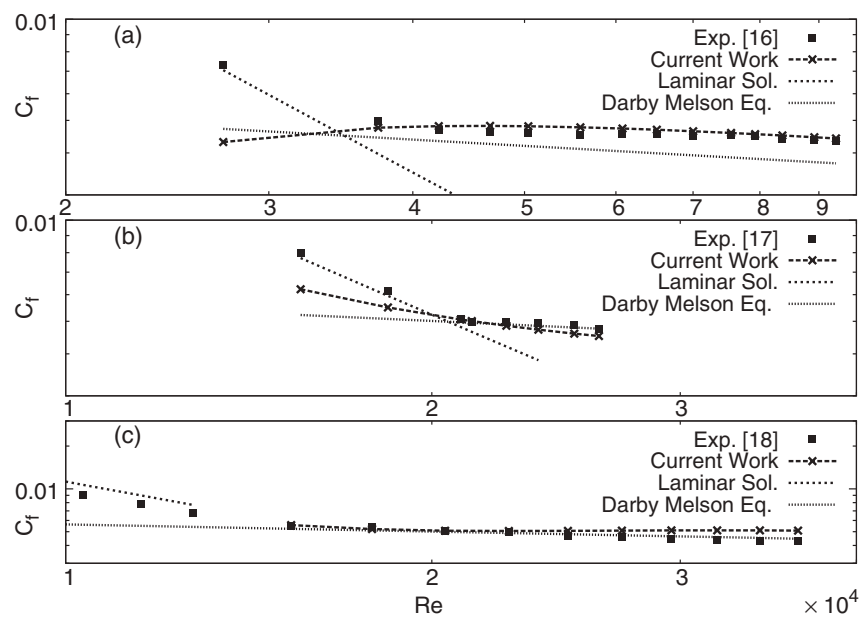

FIG. 1. Generalized Blasius equation (16), as compared with the results of the Darby-Melson formula, the laminar friction equation, and the experimental data with (a) $\mathrm{He}=2 \times 10^{6}$ from [16], (b) $\mathrm{He}=$ $7 \times 10^{5}$ from [17], and (c) $\mathrm{He}=5 \times 10^{5}$ from [18]

modified friction formula follows naturally

$$
C_{f}^{F}=8 \frac{\tau_{w}^{F}}{\rho U^{2}} \sim 8 \frac{\rho U u_{d}-\tau_{y}}{\rho U^{2}}=g(\mathrm{He}) \frac{u_{d}}{U}-8 \frac{\tau_{y}}{\rho U^{2}},
$$

where the superscript $F$ stands for the flowing part of the wall shear stress, a quantity evaluated directly from the pressure drop measurements. Inserting Eq. (11) into Eq. (15), a fundamental result arises:

$$
C_{f}^{F}=\frac{0.316}{\sqrt{2}} \sqrt{\sqrt{\frac{\mathrm{He}^{2}}{\mathrm{Re}^{4}}+\frac{4}{\mathrm{Re}}}+\frac{H e}{\mathrm{Re}^{2}}-8 \frac{\mathrm{He}}{\mathrm{Re}^{2}} .}
$$

The range of validity of the above equation is discussed in the next section; however, let us first compare the present results with some empirical works.

In Fig. 1, Eq. (16) is compared with 36 experimental points from [16-18]. Figure 1 also displays Darby and Melson's empirical law (3) for reference. The agreement is very good, with a relative error of less than $10 \%$ for all points; the empirical equation shows a maximum deviation of $15 \%$ from the experimental results. To clarify the problem discussed above, the first set of the experimental results is shown again in Fig. 2. For the sake of comparison, the results of Eqs. (14) and (16) and Blasius's formula are also shown. It is evident that

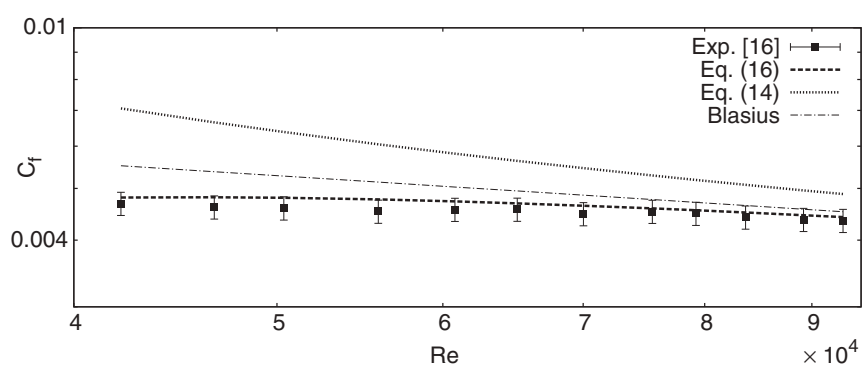

FIG. 2. Comparison of experimental results from [16] and the final friction equation (16), the friction equation without subtracting the yield stress effect (14), and the Newtonian Blasius equation. 
neglecting the last term in Eq. (16) results in an overprediction of the experimental results. Notice that, unlike the Newtonian case, it is possible to show that for some values of fixed $\mathrm{He}$, the friction formula is not a decreasing function of Re, even in the turbulent flow regime, as illustrated in Fig. 1(a). In the literature, it is common to consider that fully turbulent flow occurs for conditions past this point. However, the present results show that a combination of $\mathrm{Re}$ and $\mathrm{He}$ can result in fully turbulent flow even for lower Reynolds numbers.

We also remark that this formula reduces to the well-known Blasius equation, which is known to be a good approximation for the friction coefficient for moderately large Reynolds number, while Darby and Melson's [4] equation does not. We now turn to a brief discussion regarding the limits of validity of Eq. (16).

\section{DISCUSSION}

In this work a Blasius-type formula for the friction factor of Bingham fluids is developed. As for the Newtonian Blasius relation, the domain of validity for a fixed He extends over a limited range of Reynolds number, before wall effects need to be taken into account. For a fixed $\mathrm{He}$ and for a range of low Reynolds number, velocity fluctuations are relatively small, the inertial range is not well defined, and the viscous stress tensor is dominant over the entire flow. Under these circumstances, the present phenomenological analysis is not valid, since it depends directly on the Reynolds stress fluctuations.

For sufficiently high Re, the wall inertial stress can be defined as $\rho u_{\tau}^{2} \sim \tau_{w} \sim \tau_{w}^{F}+\tau_{y}$, so the friction velocity and the wall viscous length scale are given by

$$
u_{\tau} \equiv \sqrt{\frac{\tau_{w}^{F}+\tau_{y}}{\rho}}, \quad \delta_{v}=\frac{\mu_{b}}{\rho u_{\tau}} .
$$

Using $\tau_{w}^{F}+\tau_{y}=\rho U u_{d}$, from Eq. (11) we obtain

$$
\frac{\delta_{v}}{L} \sim \frac{1}{\operatorname{Re}}\left(\frac{U}{u_{d}}\right)^{1 / 2}=\frac{2^{1 / 4}}{\operatorname{Re}}\left(\sqrt{\frac{\mathrm{He}^{2}}{\operatorname{Re}^{4}}+\frac{4}{\operatorname{Re}}}+\frac{\mathrm{He}}{\mathrm{Re}^{2}}\right)^{-1 / 4},
$$

which reduces to the Newtonian relation when $\tau_{y}=0$. From Eq. (11) we also have

$$
\frac{\ell_{d}}{L} \sim\left(\frac{u_{d}}{U}\right)^{3}=\frac{1}{2^{3 / 2}}\left(\sqrt{\frac{\mathrm{He}^{2}}{\mathrm{Re}^{4}}+\frac{4}{\mathrm{Re}}}+\frac{\mathrm{He}}{\mathrm{Re}^{2}}\right)^{3 / 2},
$$

where $\delta_{v} \equiv \delta_{v}(\mathrm{He}, \mathrm{Re})$ and $\ell_{d} \equiv \ell_{d}(\mathrm{He}, \mathrm{Re})$. Let $s$ denote the characteristic size of the eddies transporting momentum over $\mathcal{W}$ and let $a_{\mathrm{He}} \delta_{v}$ denote the characteristic thickness of the viscous surface, where $a_{\mathrm{He}}$ is an $O(1)$ constant that needs to be estimated from experiments (in turbulent Newtonian flows, $a_{0}$ is typically 5 ; see [8]).

In our moderate-Reynolds-number scenario, it results from (17) and (18) that for a fixed He, as Re increases, eddies in the transitional region become both slower and smaller, just as well as the wall viscous length scale. The ratio $H$ between the thickness of the viscous layer $a_{\mathrm{He}} \delta_{v}$ and the transitional

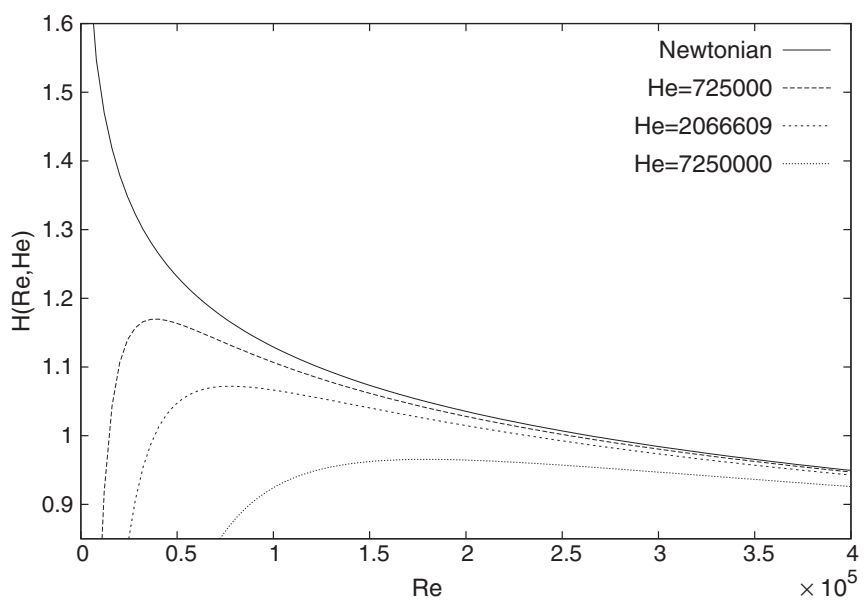

FIG. 3. Plot of $H \equiv \delta_{v}^{(n)} / \ell_{d}^{(n)}$ versus Re, for some fixed values of He. Notice that $H<1$ for $\operatorname{Re} \sim 3.5 \times 10^{5}$, which is compatible with Blasius's range of validity. Notice also that for $\mathrm{He}=7.25 \times 10^{6}$, the ratio $H$ is never greater than 1 .

Kolmogorov scale $\ell_{d}$ satisfies

$$
H(\mathrm{Re}, \mathrm{He}) \equiv \frac{a_{\mathrm{He}} \delta_{v}}{\ell_{d}} \sim \frac{2^{7 / 4}}{\mathrm{Re}}\left(\sqrt{\frac{\mathrm{He}^{2}}{\mathrm{Re}^{4}}+\frac{4}{\mathrm{Re}}}+\frac{\mathrm{He}}{\mathrm{Re}^{2}}\right)^{-7 / 4} .
$$

For a range of Re so that $s \sim \ell_{d}<a_{\mathrm{He}} \delta_{v}$, i.e., $H>1$, the flow near the wall is well described by the phenomenology of the preceding section and (15) is a good approximation. However, if $\ell_{d} \geqslant a_{\mathrm{He}} \delta_{v}$, i.e., $H<1$, then transitional eddies become too large to get absorbed in the viscous layer, so the present phenomenology breaks down and wall properties have to be considered. Notice that if we consider that $a_{\mathrm{He}}$ remains $O(1)$, then, for sufficiently large $\mathrm{He}$, the phenomenological analysis here presented is no longer valid for any value of Reynolds number. In fact, Figs. 3 and 4 show the behavior of the ratio function $H$ by assuming that $a_{\mathrm{He}}=a_{0}=5$ and we can see that $H<1$ for all values of $\mathrm{Re}$, and $\mathrm{He} \lesssim 0.8 \times$ $10^{7}$. The assumption that $a_{\mathrm{He}}$ remains $O(1)$ is crucial for the

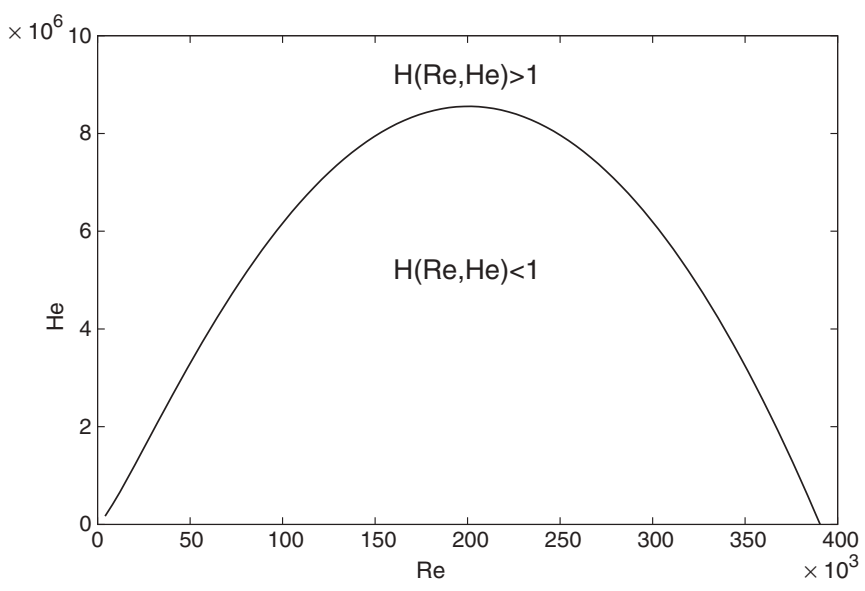

FIG. 4. Level curve $H \equiv 1$. We expect the domain of validity of Eq. (16) to be in a region similar to the region where $H<1$. 
analysis above. This is a consideration that deserves further investigation. We remark, however, that, as discussed in [19], Darby and Melson's empirical expression is considered to be valid in a range where $1000 \leqslant \mathrm{He} \leqslant 6.6 \times 10^{7}$, and $\operatorname{Re}_{B} \leqslant$ $3.4 \times 10^{5}$, which is compatible with the constraints displayed in Figs. 3 and 4.

\section{CONCLUSION}

The present work has developed Eq. (16), a friction factor formula for Bingham fluid flows. The arguments were dimensional, in the spirit of Kolmogorov's analysis, and further resorted to a phenomenological closure relation for the Reynolds' stress, similar to that proposed by Gioa and Chakraborty in [5]. We have also derived limits of validity of a presently proposed equation through a phenomenological argument first introduced in [6]. The limits are compatible with previous empirical Bingham friction relations, such as that proposed by Darby and Melson in [4]. Equation (16) is derived from Eq. (14) through a correction term that takes into account a discrepancy between the latter equation and the experimental data observed in the literature. Pressure drop discrepancies in Bingham flows have been observed before, as remarked in [15]; this continues to be a challenging theoretical and experimental issue. Nonetheless, Eq. (16) has shown excellent agreement with the experimental data for an extensive range of $\mathrm{Re}$ and $\mathrm{He}$ and, moreover, similar arguments may be extended to other types of purely viscous yielding flows, such as Herschel-Bulkley fluids. This extension is currently under investigation, but preliminary results show remarkable agreement with experimental data.

\section{ACKNOWLEDGMENTS}

A.P.S.F. is grateful to the Brazilian National Research Council (CNPq) for support through the award of a Research Fellowship (Grant No. 305338/2014-5). The work was financially supported by $\mathrm{CNPq}$ through Grant No. 477293/2011-5 and by the Rio de Janeiro Research Foundation through Grants No. E-26/102.937/2011 and No. E26/010.001275/2016 (Pronex).
[1] P. R. H. Blasius, Das aehnlichkeitsgesetz bei reibungsvorgangen in flüssigkeiten, Forschungsheft 131, 1 (1913).

[2] J. Nikuradse, Gesetzmäßigkeit der turbulenten strömung in glatten rohren, Forsch. Arb. Ing. Wes. 356 (1932) (English translation NACA TT F-10, p. 359).

[3] D. W. Dodge and A. B. Metzner, Turbulent flow of nonnewtonian systems, AIChE J. 5, 189 (1959).

[4] R. Darby and J. Melson, How to predict the friction factor for flow of Bingham plastics, Chem. Eng. 28, 59 (1981).

[5] G. Gioia and P. Chakraborty, Turbulent Friction in Rough Pipes and the Energy Spectrum of the Phenomenological Theory, Phys. Rev. Lett. 96, 044502 (2006).

[6] H. R. Anbarlooei, D. O. A. Cruz, F. Ramos, and A. P. Silva Freire, Phenomenological Blasius-type friction equation for turbulent power-law fluid flows, Phys. Rev. E 92, 063006 (2015).

[7] A. N. Kolmogorov, The local structure of turbulence in incompressible viscous fluid for very large Reynolds numbers, Proc. Math. Phys. Sci. 434, 9 (1991).

[8] S. B. Pope, Turbulent Flows (Cambridge University Press, Cambridge, 2000).

[9] U. Frisch, Turbulence: The Legacy of A. N. Kolmogorov (Cambridge University Press, Cambridge, 1995).

[10] B. Knight and L. Sirovich, Kolmogorov Inertial Range for Inhomogeneous Turbulent Flows, Phys. Rev. Lett. 65, 1356 (1990).

[11] J. Peixinho, C. Nouar, C. Desaubry, and B. Théron, Laminar transitional and turbulent flow of yield stress fluid in a pipe, J. Non-Newtonian Fluid Mech. 128, 172 (2005).
[12] B. Güzel, T. Burghelea, I. A. Frigaard, and D. M. Martinez, Observation of laminar-turbulent transition of a yield stress fluid in Hagen-Poiseuille flow, J. Fluid Mech. 627, 97 (2009).

[13] I. A. Frigaard, S. D. Howison, and I. J. Sobey, On the stability of Poiseuille flow of a Bingham fluid, J. Fluid Mech. 263, 133 (1994).

[14] M. P. Escudier and F. Presti, Pipe flow of a thixotropic liquid, J. Non-Newtonian Fluid Mech. 62, 291 (1996).

[15] G. M. Oliveira, C. O. R. Negro, and A. T. Franco, Pressure transmission in Bingham fluids compressed within a closed pipe, J. Non-Newtonian Fluid Mech. 169-170, 121 (2012).

[16] A. Bartosik, Application of rheological models in prediction of turbulent slurry flow, Flow Turbul. Combust. 84, 277 (2010).

[17] D. J. Hallbom, Pipe flow of homogeneous slurry, Ph.D. thesis, University of British Columbia, 2008.

[18] A. P. Poloski, M. L. Bonebrake, A. M. Casella, M. D. Johnson, P. J. MacFarlan, J. J. Toth, H. E. Adkins, J. Chun, K. M. Denslow, M. L. Luna, and J. M. Tingey, Deposition velocities of non-Newtonian slurries in pipelines: Complex simulant testing, U.S. DOE Report No. PNNL-18316, prepared by Pacific Northwest National Laboratory, 2009 (unpublished).

[19] R. Chhabra and J. Richardson, Non-Newtonian Flow and Applied Rheology: Engineering Applications (ButterworthHeinemann, Oxford, 2011). 